\title{
O ARGUMENTO DO MILAGRE COMETE A FALÁCIA DA TAXA-BASE? APRESENTAÇÃO, ESTADO DA ARTE E QUESTÕES DE FORMALIZAÇÃO
}

\author{
Does the No-Miracles Argument commit the Base Rate Fallacy? Presentation, State of the Art \\ and Formalization Difficulties
}

Pedro Bravo de Souza *

Resumo: Objetivamos discutir a crítica, avançada por Colin Howson em Hume's problem, segundo a qual o argumento do milagre (doravante, AM) comete a falácia da taxa-base. Por falácia da taxa-base, entende-se a negligência do valor da probabilidade prévia de determinada hipótese ou teoria $T, \mathrm{P}(\mathrm{T})$. Por sua vez, em uma de suas versões, AM assere que apenas assumindo que uma teoria científica madura $T$ é aproximadamente verdadeira não faz de seu sucesso preditivo um milagre. Formalizado probabilisticamente, Howson argumenta que a conclusão de AM, a probabilidade de que a teoria $T$ seja aproximadamente verdadeira é grande, apenas se segue se assumirmos para $T$ um valor não-negligenciável. Tal crítica de Howson despertou duas categorias de reação na literatura especializada: supondo que AM comete a falácia da taxa base, alguns autores propuseram abandonar $\mathrm{AM}$ enquanto um argumento para a tese epistemológica do realismo científico, ao passo que outros defenderam que apenas uma versão de $\mathrm{AM}$ comete a falácia da taxa-base; defendendo que AM não comete a falácia da taxa base, certos autores alegaram alguns problemas na formalização probabilística de AM, enquanto outros buscaram refinar tal formalização de modo que a nova evite a falácia em questão. Tendo apresentado esse estado da arte, investigaremos se a formalização probabilística do argumento do milagre é razoável. Discutiremos se ela acomoda a natureza de inferência à melhor explicação pressuposta no AM. Por fim, indicaremos, brevemente, algumas dificuldades para a crítica de Howson ao assumir-se que AM instancia, na verdade, uma abdução Peirceana.

Palavras-chave: Filosofia das Ciências; Argumento do Milagre; Realismo; Probabilidade; Inferência à Melhor Explicação.

\begin{tabular}{|c|c|c|c|c|c|}
\hline intuitio & $\begin{array}{c}\text { ISSN } \\
1983-4012\end{array}$ & Porto Alegre & Vol.11 $-\mathrm{N}^{\mathrm{o}} .1$ & $\begin{array}{l}\text { Julho } \\
2018\end{array}$ & p.46-64 \\
\hline
\end{tabular}


O argumento do milagre comete a falácia da taxa-base? Apresentação, estado da arte e questões de formalização

Abstract: We aim to discuss Colin Howson's objection, presented in his book Hume's problem, according to which the no-miracles argument (henceforth, NMA) commits the base rate fallacy. By base rate fallacy, we mean the prior probability value of a particular hypothesis or theory $T, \mathrm{P}(\mathrm{T})$, neglect. In turn, in one of its versions, NMA asserts that only assuming that a mature scientific theory $T$ is approximately true does not make its predictive success a miracle. When probabilistically formalized, Howson argues that NMA conclusion, the probability that a theory $T$ is approximately true is large, only follows if we assume for $T$ a non-negligible value. Howson's objection aroused two categories of reaction in the specialized literature: supposing that NMA commits the base rate fallacy, some authors proposed to abandon NMA as an argument for scientific realism epistemological thesis, while others argued that only one version of NMA commits the base rate fallacy; arguing that NMA does not commit the base rate fallacy, some authors have pointed ou some difficults in NMA probabilistic formalization, while others have sought to refine such formalization so that the new one would avoid the fallacy in question. After presenting this state of the art, we will investigate whether or not the probabilistic formalization of the no-miracles argument is reasonable. We will discuss whether or not it accommodates the nature of inference to the best explanation presupposed in NMA. Finally, we will briefly outline some difficulties for Howson's objection when it is assumed that NMA instantiates, rather, a Peircean abduction.

Keywords: Philosophy of Sciences; No-Miracle Argument; Realism; Probability; Inference to the Best explanation.

* Pedro Bravo de Souza é mestrando em Filosofia pela UNESP-Marília, sob a orientação do Prof. Dr. Marcos Antonio Alves e com financiamento da FAPESP (Processo $n^{\circ}$ 2016/03251-2). Correio eletrônico: pedrobravodesouza@hotmail.com.

The debate between scientific realists and anti-realists is one of the classics of philosophy of science, comparable to a soccer match between Brazil and

Argentina.

Jan Sprenger

\section{Introdução}

O uso do aparato probabilístico em filosofia tem se intensificado no decorrer das últimas décadas e, assim, incidido em seus mais diversos temas e problemas. Para apenas ficarmos em alguns exemplos, discussões sobre graus de crença, confirmação, percepção e cognição são hoje debatidas também recorrendo-se ao cálculo de probabilidades e, principalmente, à sua interpretação epistêmica ou Bayesiana: o valor da probabilidade de uma proposição $P$ mede o quão confiante um agente está na verdade de $P .{ }^{1}$ Nesse contexto, o presente artigo visa discutir o uso de conceitos de probabilidade na

1 Além dos textos deste dossiê, ver, para uma excelente introdução em português ao uso da interpretação Bayesiana em epistemologia, bem como discussões sobre graus de crença e confirmação, o artigo e as referências de Neiva

\begin{tabular}{|c|c|l|l|l|l|}
\hline intuitio & $\begin{array}{c}\text { ISSN } \\
1983-4012\end{array}$ & Porto Alegre & Vol.11- $\mathrm{N}^{\circ} .1$ & $\begin{array}{l}\text { Julho } \\
2018\end{array}$ & p.46-64 \\
\hline
\end{tabular}


O argumento do milagre comete a falácia da taxa-base? Apresentação, estado da arte e questões de formalização

polêmica disputa entre realistas e antirrealistas científicos. Mais precisamente, debateremos uma crítica de Colin Howson $(2000 ; 2013)$ a uma formalização probabilística do argumento do milagre.

$\mathrm{O}$ argumento do milagre (doravante, AM) é considerado por muitos filósofos das ciências um dos principais argumentos a favor da tese epistemológica do realismo científico: teorias científicas são aproximadamente verdadeiras. Em uma de suas versões, AM expressa que apenas assumindo que uma teoria científica madura $T$ é aproximadamente verdadeira não faz de seu sucesso preditivo um milagre. Considerando uma formalização probabilística de AM (chamemo-la de AMP), Howson recentemente criticou AM atribuindo-lhe a falácia da taxa-base, ou seja, a negligência do valor da probabilidade prévia de determinada teoria $T$.

Essa crítica de Howson vem recebendo, pelo menos, duas categorias de reação na literatura especializada: supondo que AM comete a falácia da taxa-base, alguns autores propuseram abandonar AM enquanto um argumento para a tese epistemológica do realismo científico, ao passo que outros defenderam que apenas uma versão de AM comete a falácia da taxa-base, limitando, então, o escopo da crítica de Howson; defendendo que AM não comete a falácia da taxa base, certos autores alegaram alguns problemas na formalização probabilística de AM, enquanto outros buscaram refinar AMP de modo que a nova formalização evite a falácia em questão.

O objetivo geral deste artigo é discutir a crítica de Howson ao AM. Para tanto, apresentaremos AM na próxima seção, bem como a falácia da taxa-base e como Howson a atribui ao AM. Em seguida, introduzimos, rapidamente, o estado da arte em relação a essa crítica sem, no entanto, posicionarmo-nos em relação a cada uma das respostas a Howson. Num terceiro momento, debateremos a razoabilidade de AMP tendo em mente os desiderata de fecundidade e similaridade de uma formalização. Por fim, indicaremos algumas dificuldades para a crítica de Howson, admitindo que AM instancia uma inferência abdutiva.

Antes de iniciarmos nossas considerações, faz-se necessário comentar, rapidamente, apenas alguns dos elementos do Bayesianismo que são importantes para este artigo. De modo geral, pode-se definir Bayesianismo como uma corrente teórica decorrente da adoção de três teses básicas (Easwaran, 2011):

Gradualismo: agentes possuem graus de crença para os quais valores numéricos podem ser atribuídos; Probabilismo: os graus de crença de um agente racional devem obedecer ao cálculo de probabilidades; Revisão pela condicionalização: um agente racional deve, quando adquire nova evidência, atualizar os seus graus de crença conforme regras de condicionalização.

(2015). Para um texto que discuta percepção e Bayesianismo, ver Rescorla (2015). Para textos que discutam cognição e probabilidade, ver Rescorla (2009) e Seriès (2016).

\begin{tabular}{|c|c|l|l|l|l|}
\hline intuitio & $\begin{array}{c}\text { ISSN } \\
1983-4012\end{array}$ & Porto Alegre & Vol.11- $\mathrm{N}^{\mathrm{o}} .1$ & $\begin{array}{l}\text { Julho } \\
2018\end{array}$ & p.46-64 \\
\hline
\end{tabular}


O argumento do milagre comete a falácia da taxa-base? Apresentação, estado da arte e questões de formalização

Três comentários sobre as teses mencionadas e outros quatro acerca de demais aspectos do Bayesianismo para nós importantes. (1) Graus de crença expressariam mediante números quão confiantes determinados agentes estão em relação à determinada proposição. Para efeito de ilustração, considere que estou um tanto quanto certo que "o realismo científico é verdadeiro". Assim, meu grau de crença em tal proposição poderia assumir, digamos, o valor $0.8 .^{2}$ (2) A formalização do cálculo de probabilidade suposto pela tese do probabilismo é, em geral, semelhante àquela fornecida por Kolmogorov (1950), com a diferença de que os relata da função de probabilidade são proposições e não eventos. (3) A revisão pela condicionalização visa restringir a atualização de graus de crença através do tempo. Uma das regras mais conhecidas para fazê-lo é a condicionalização estrita. Segundo ela, quando um agente racional adquire uma nova evidência $E$, a nova probabilidade de uma hipótese $H$ deve ser igual à probabilidade condicional anterior de $H$ dado $E$. Formalmente, tem-se que: $\mathrm{P}_{\text {nova }}(\mathrm{H})=\mathrm{P}(\mathrm{H} \mid \mathrm{E})$.

(4) O nome Bayesianismo advém do amplo uso pelos autores que adotam as teses indicadas do teorema de Bayes, creditado, corretamente ou não, ao reverendo Thomas Bayes (1702-1761). Em uma de suas versões, tal teorema é assim expresso: $\mathrm{P}(\mathrm{H} \mid \mathrm{E})=\mathrm{P}(\mathrm{H}) \times \mathrm{P}(\mathrm{E} \mid \mathrm{H}) / \mathrm{P}(\mathrm{E})$, em que $\mathrm{P}(\mathrm{H} \mid \mathrm{E})$ expressa a probabilidade posterior de uma hipótese ou teoria $H, \mathrm{P}(\mathrm{H})$ simboliza a probabilidade prévia de $H, \mathrm{P}(\mathrm{E})$ expressa a probabilidade prévia de uma evidência $E$, e, finalmente, $\mathrm{P}(\mathrm{E} \mid \mathrm{H})$ simboliza a probabilidade de $E$ dado que $H$. (5) Bayesianos que adotam somente as teses básicas acima são conhecidos como Bayesianos subjetivos. Bayesianos que adotam algum princípio que visa relacionar probabilidades objetivas e probabilidades epistêmicas são Bayesianos moderados. Por fim, Bayesianos que defendem algum princípio para restringir probabilidades prévias são Bayesianos objetivos (ver apêndice 1). (6) Por si só o aparato Bayesiano não permite concluir que, se a probabilidade de determinada hipótese $H$ é maior que determinado limiar $x$, então podemos aceitar racionalmente $H$; há, no entanto, princípios que procuram estabelecer justamente essa conexão. Dentre eles, destaca-se a tese Lockeana, segundo a qual um agente $A$ racionalmente aceita $H$ se, e somente se, o grau de crença de $A$ em $H$ é maior a algum limiar $x$. (7) Mediante as noções expostas, é possível definir a relação de confirmação incremental entre uma hipótese científica $H$ e uma evidência $E$ : uma evidência $E$ confirma uma hipótese $H$ se, e somente se, $\mathrm{P}(\mathrm{H} \mid$ E) > P(H), uma das cláusulas da teoria Bayesiana da confirmação. ${ }^{3}$

\footnotetext{
2 Dizer que um grau de crença assume o valor 0.8 poderia supor que há exclusivamente graus precisos de crença, o que não é, necessariamente, o caso. Ora, há propostas que permitem atribuir valores imprecisos a proposições, isto é, valores pertencentes a um subintervalo de $[0,1]$. Como apenas pressuporemos o modelo de graus precisos de crença neste artigo, apenas indicamos ao leitor interessado a seção 1.2.3 de Weisberg (2011).

3 Essa apresentação do Bayesianismo é, embora atenda a nossos objetivos, certamente simplificada. Para detalhes de cada um dos comentários feitos, ver Weisberg (2011), talvez um dos mais completos textos sobre Bayesianismo.
}

\begin{tabular}{|c|c|l|l|l|l|}
\hline intuitio & $\begin{array}{c}\text { ISSN } \\
1983-4012\end{array}$ & Porto Alegre & Vol.11- $\mathrm{N}^{\mathrm{o}} .1$ & $\begin{array}{l}\text { Julho } \\
2018\end{array}$ & p.46-64 \\
\hline
\end{tabular}




\section{AM, a falácia da taxa-base e a crítica de Howson}

Conforme Bas van Fraassen (1980: 39), AM é considerado como o argumento derradeiro para o realismo científico. Mais precisamente, AM permite concluir a tese epistemológica do realismo científico: uma ou a(s) teoria(s) científica(s) madura(s) são aproximadamente verdadeiras. É importante salientar que um realista científico pode assumir, porém, outras teses, como a tese metafísica segundo a qual o que as teorias científicas descrevem é independente de nossos pensamentos, ou a tese semântica de que mesmo os termos inobserváveis utilizados nas teorias científicas possuem referentes factuais (Psillos, 1999, p. xvii). AM restringe-se, portanto, a defender apenas uma das teses do realismo científico.

Originalmente, AM dizia apenas que: "O argumento positivo para o realismo é que ele é a única filosofia que não faz do sucesso da ciência um milagre" (Putnam, 1975: 73; tradução nossa). ${ }^{4}$ Em resposta a diversas críticas, AM foi reformulado. Howson (2000: 36), em particular, apresenta a seguinte versão:

\section{(AMH) Argumento do Milagre conforme Howson}

P1: Se uma teoria $T$ prediz independentemente um dado observacional $E$ e $T$ não é aproximadamente verdadeira, então o seu acordo com $E$ deve ser acidental.

P2: $\quad$ Os dados de $E$ são tais que um acordo acidental de $T$ com eles é extremamente improvável.

P3: A minúscula probabilidade de $T$ estar em acordo com $E$ sem ser aproximadamente verdadeira é extraordinariamente tão pequena de modo que podemos rejeitar a hipótese segundo a qual há um acordo acidental.

Concl: $T$ é aproximadamente verdadeira.

Antes de comentarmos $\mathrm{AMH}$, convém dizer que ele é criticado por Howson (2000) à medida que, para tal autor, AMH poderia fornecer uma justificação para a inferência indutiva de que determinada teoria $T$ é aproximadamente verdadeira. ${ }^{5}$ Ao argumentar que esse não é o caso - tampouco é o caso de outras propostas que se dirigem ao problema da indução -, Howson vai pavimentando o caminho para sua proposta Bayesiana.

Feita essa contextualização, voltemo-nos a AMH. Sua primeira premissa (P1) afirma que, se uma teoria $T$ prediz de modo independente $E$, isto é, sem ter sido elaborada para acomodar $E$, e $T$ não é aproximadamente verdadeira, então seu acordo com $E$ deve ser um resultado do acaso. Para efeito de

4 No original: "The positive argument for realism is that it is the only philosophy that doesn't make the success of science a miracle".

5 Parece-nos, porém, que AMH dificilmente não estaria no escopo do argumento de Hume quanto à indução: como justificar que um milagre é extremamente improvável senão recorrendo a experiências passadas e, assim, pressupondo alguma forma de uniformidade da natureza?

\begin{tabular}{|c|c|l|l|l|l|}
\hline intuitio & $\begin{array}{c}\text { ISSN } \\
1983-4012\end{array}$ & Porto Alegre & Vol.11- $\mathrm{N}^{\mathrm{o}} .1$ & $\begin{array}{l}\text { Julho } \\
2018\end{array}$ & p.46-64 \\
\hline
\end{tabular}


O argumento do milagre comete a falácia da taxa-base? Apresentação, estado da arte e questões de formalização

ilustração, se a teoria que predisse independentemente o retorno do cometa Halley não é aproximadamente verdadeira, então seu acordo com o dado observado ocorreu acidentalmente.

Por sua vez, a segunda premissa (P2) afirma que o dado observacional $E$ é de tal modo preciso que a probabilidade de $T$ estar em acordo com ele acidentalmente é extremamente pequena. Considerando o sucesso preditivo do valor do momento magnético ${ }^{6}$ de um elétron (a predição era $1.00115965246 \pm$ 0.00000000020 e o valor observado foi $1.00115965221 \pm 0.00000000003)$, parece plausível defender a hipótese segundo a qual um acordo acidental nesse caso seria altamente improvável (Howson, 2000: 37).

Enfim, a terceira premissa (P3) afirma que probabilidades tais como ilustradas em P2 são tão ínfimas que podemos rejeitar a hipótese de um acordo acidental. Alternativamente, elas são tão improváveis que sua ocorrência assemelhar-se-ia a um milagre. Ora, milagres são extremamente improváveis. Não há, por conseguinte, um acordo acidental. Pode-se concluir, então, que $T$ é aproximadamente verdadeira. Em geral, "aproximadamente verdadeiro", em vez de simplesmente "verdadeiro", consta em AM em função de evidências na história das ciências em que uma teoria com grande sucesso preditivo se revelou não apenas superada por uma teoria posterior, senão falseada em determinados domínios. Esse é o caso, notadamente, da mecânica newtoniana em face da teoria da relatividade geral de Einstein (Worrall, 1989: 104).

Após criticar cada uma das premissas acima, Howson (2000: 55-57; 2013) defende que a conclusão de $\mathrm{AMH}$ apenas decorre de suas premissas ao assumirmos um valor não-negligenciável (maior que 0.2) para a probabilidade prévia de que $T$ é aproximadamente verdadeira: AMH comete, assim, a falácia da taxa-base. ${ }^{7}$ Em outras palavras, AMH apenas consegue sustentar sua conclusão se já pressupõe uma probabilidade não muito baixa para a verdade aproximada da teoria $T$. Um antirrealista poderia, então, criticar um realista que se apoie em AMH alegando que ele comete uma petição de princípio ao assumir justamente o que está em disputa: a verdade aproximada de $T$.

Para bem compreendermos como Howson apresenta essa crítica, é necessário antes apresentarmos AMH em sua versão probabilística. Ao longo de nossas considerações, assumiremos que uma probabilidade $x$ é grande se $x>0.85$, e $x$ é não-negligenciável se $x>0.2$. Seja "a teoria $T$ é bem-sucedida preditivamente" simbolizado por $S$ e "a teoria $T$ é aproximadamente verdadeira" simbolizado por $V$, temos que:

6 Grosso modo, o momento magnético é a medida de intensidade da fonte magnética de um corpo, como um imã, a Terra ou, no caso exemplificado, um elétron.

7 Dawid \& Hartmann (2017) afirmam que Howson inaugurou uma nova linha de crítica ao AM ao atacar sua inferência e não, como vários autores fazem, cada uma de suas premissas. Esse, porém, não é o caso. Musgrave (1988) já havia criticado a inferência de AM: considerando AM como um entimema, a premissa que lhe falta seria o princípio metafísico de que a melhor explicação para um fato é ela mesma ser verdadeira, o que, para Musgrave, é obviamente falso.

\begin{tabular}{|c|c|l|l|l|l|}
\hline intuitio & $\begin{array}{c}\text { ISSN } \\
1983-4012\end{array}$ & Porto Alegre & Vol.11- $\mathrm{N}^{\mathrm{o}} .1$ & $\begin{array}{l}\text { Julho } \\
2018\end{array}$ & p.46-64 \\
\hline
\end{tabular}




\section{(AMP) Argumento do Milagre Probabilístico}

$\mathrm{P} 1 *: \quad \mathrm{P}(\mathrm{S} \mid \mathrm{V})$ é grande.

$\mathrm{P} 2 *: \quad \mathrm{P}(\mathrm{S} \mid \neg \mathrm{V})$ é extremamente pequena.

Concl: $\mathrm{P}(\mathrm{V} \mid \mathrm{S})$ é grande.

Howson (2013: 205) afirma não somente que a formalização acima é natural, como implícita no significado de milagre em AMH como um evento extremamente improvável. Assumindo a interpretação epistêmica ou Bayesiana de probabilidade, isto é, P(A) significa o quão confiante determinado agente está na verdade de determinada proposição $A$, clarifiquemos as premissas acima. P1* expressa que a probabilidade do sucesso preditivo de uma teoria $T$, supondo que $T$ é aproximadamente verdadeira, é grande. Por sua vez, $\mathrm{P} 2 *$ afirma que a probabilidade do sucesso preditivo da teoria $T$, supondo que não é o caso que $T$ seja aproximadamente verdadeira, é extremamente pequena (próxima a 0). Conclui-se, então, que a probabilidade de que a teoria $T$ seja aproximadamente verdadeira, supondo seu sucesso preditivo, é grande.

De modo a perceber como a conclusão apenas decorre se $\mathrm{P}(\mathrm{V})$ assumir algum valor nãonegligenciável (maior que 0.2), exemplificaremos a falácia da taxa-base por meio de um de seus exemplos mais conhecidos: o Teste da Escola Médica de Harvard (doravante, TH). Realizado por Casscells et al. (1978), TH consistia em um problema hipotético dirigido a estudantes de medicina da Universidade de Harvard. Seu enunciado pode ser assim apresentado: suponha que um teste para diagnosticar uma doença $D$ tenha dois resultados possíveis: positivo e negativo. A probabilidade de que o teste resulte negativo quando um sujeito examinado tem a doença (falso negativo) é 0 , e a probabilidade de que o teste resulte positivo quando um sujeito examinado não tem a doença (falso positivo) é 0.05. Finalmente, considere que, em determinada população, a probabilidade de alguém ter a doença $D$ é 1/1000 (0.001). Questiona-se: se um certo sujeito dessa população obteve um teste positivo, qual a probabilidade de que ele tenha a doença $D$ ?

Formalizemos, em primeiro lugar, os dados acima antes de responder à questão enunciada. Assuma que $E$ represente "o resultado do teste foi positivo" e que $H$ represente "o sujeito tem a doença". Assim, $\mathrm{P}(\mathrm{H})$ expressa a probabilidade prévia de alguém ter a doença (valor 0.001$) ; \mathrm{P}(\mathrm{E} \mid \mathrm{H})$ corresponde à probabilidade de que o resultado do teste foi positivo, dado que o sujeito tem a doença (valor 1, dado que o valor do falso negativo é zero); $\mathrm{P}(\mathrm{E} \mid \neg \mathrm{H})$ simboliza a probabilidade de que o resultado do teste foi positivo, dado que o sujeito não tem a doença (valor 0.05); $\mathrm{P}(\neg \mathrm{H})$ representa a probabilidade de que o sujeito não tenha a doença (valor 0.999) e, finalmente, $\mathrm{P}(\mathrm{H} \mid \mathrm{E})$ corresponde ao que queremos saber, ou seja, a probabilidade de um sujeito tenha a doença, dado que o teste foi positivo.

\begin{tabular}{|c|c|c|c|c|c|}
\hline intuitio & ISSN & Porto Alegre & Vol.11- $\mathrm{N}^{\circ} .1$ & $\begin{array}{l}\text { Julho } \\
2018\end{array}$ & p.46-64 \\
\hline
\end{tabular}


O argumento do milagre comete a falácia da taxa-base? Apresentação, estado da arte e questões de formalização

Representemos agora tais dados em um modelo de retirada de bolas de uma urna (Howson, 2000: 53). Nela, há apenas bolas brancas e pretas inscritas ou com o número 1 ou com o número 0 . Uma bola preta é uma pessoa portadora da doença, e uma bola branca é uma pessoa sem doença. Toda bola com o número 1 é uma pessoa cujo teste foi positivo, e toda bola com o número 0 é uma pessoa cujo teste foi negativo. Se há 1000 bolas na urna, apenas uma deve ser uma bola preta, dado que $\mathrm{P}(\mathrm{H})=0.001$. Como $\mathrm{P}(\mathrm{E} \mid \mathrm{H})=1$, essa mesma bola está marcada com o número 1. Recordando que a probabilidade de que o resultado do teste foi positivo, dado que o sujeito não tem a doença, $\mathrm{P}(\mathrm{E} \mid \neg \mathrm{H})=0.05,5 \%$ das 999 bolas restantes também estarão com o número 1, ou, aproximadamente, 50. Portanto, 51 bolas estão inscritas com o número 1, mas apenas uma é preta. Logo, a probabilidade de que uma pessoa tenha a doença, dado que o teste foi positivo, é de $1 / 51=0.019$, ou, aproximadamente, $2 \%$.

Os estudantes aos quais tal problema foi dirigido responderam 95\%, considerando apenas os valores do falso positivo e falso negativo. Nota-se que eles ignoraram a taxa base ou probabilidade prévia da doença, $\mathrm{P}(\mathrm{H})$, cujo valor é crucial para a resolução do problema. Com efeito, em vez de uma grande probabilidade de que o sujeito tenha a doença, levando-se em conta $\mathrm{P}(\mathrm{H})$ obtemos apenas $2 \%$ de probabilidade. Graças a esse experimento, raciocinar não se levando em conta tal expressão ficou conhecido como a falácia da taxa-base. Ora, conforme Howson (2000; 2013), o mesmo erro de tais estudantes estaria presente em AMP e, consequentemente, em AM. Esquematicamente, teríamos que:

(AMP) Argumento do Milagre Probabilístico

P1*: $\quad \mathrm{P}(\mathrm{S} \mid \mathrm{V})$ é grande.

$\mathrm{P} 2 *: \quad \mathrm{P}(\mathrm{S} \mid \neg \mathrm{V})$ é extremamente pequena.

Concl: $\quad \mathrm{P}(\mathrm{V} \mid \mathrm{S})$ é grande.

\section{(TH) Teste da Escola Médica de Harvard}

P1*: $\quad \mathrm{P}(\mathrm{E} \mid \mathrm{H})$ é grande (1).

$\mathrm{P} 2 *$ : $\quad \mathrm{P}(\mathrm{E} \mid \neg \mathrm{H})$ é extremamente pequena (0.05).

Concl: $\quad \mathrm{P}(\mathrm{H} \mid \mathrm{E})$ é grande. $*$

Esquema 1: Comparação entre AMP e TH.

Conforme TH exemplifica, apenas os valores de $\mathrm{P}(\mathrm{E} \mid \mathrm{H})$ e $\mathrm{P}(\mathrm{E} \mid \neg \mathrm{H})$ são insuficientes para determinar o valor de $\mathrm{P}(\mathrm{H} \mid \mathrm{E}) .{ }^{8}$ Ainda pior, dependendo do valor de $\mathrm{P}(\mathrm{H})$, e assumindo valores de $\mathrm{P}(\mathrm{E} \mid$ $\mathrm{H})$ e $\mathrm{P}(\mathrm{E} \mid \neg \mathrm{H})$ semelhantes aos valores $\mathrm{P}(\mathrm{S} \mid \mathrm{V})$ e $\mathrm{P}(\mathrm{S} \mid \neg \mathrm{V})$ de $\mathrm{AMP}$, não se segue que $\mathrm{P}(\mathrm{H} \mid \mathrm{E})$ ou $\mathrm{P}(\mathrm{V} \mid$ S) seja grande. Na verdade, como vimos, $\operatorname{se} \mathrm{P}(\mathrm{H})=0.001, \mathrm{P}(\mathrm{H} \mid \mathrm{E}) \approx 0.02$ ! Retornando ao modelo da urna, se $\mathrm{P}(\mathrm{H})=0.1, \mathrm{P}(\mathrm{H} \mid \mathrm{E}) \approx 0.68$. Se $\mathrm{P}(\mathrm{H})=0.2, \mathrm{P}(\mathrm{H} \mid \mathrm{E}) \approx 0.83$. Se $\mathrm{P}(\mathrm{H})=0.3, \mathrm{P}(\mathrm{H} \mid \mathrm{E}) \approx 0.9$.

Nesse sentido, um realista que defenda AMP teria que assumir um valor de $\mathrm{P}(\mathrm{V})$ pelo menos acima de 0.2 para concluir que $\mathrm{P}(\mathrm{V} \mid \mathrm{S})$ é grande, cometendo, assim, uma petição de princípio. Nessas

8 Que os valores de $\mathrm{P}(\mathrm{E} \mid \mathrm{H})$ e $\mathrm{P}(\mathrm{E} \mid \neg \mathrm{H})$ são insuficientes para determinar o valor de $\mathrm{P}(\mathrm{H} \mid \mathrm{E})$ é facilmente percebido na forma a seguir do teorema de Bayes (seguirei, contudo, usando o modelo da urna para ser mais didático): $\mathrm{P}(\mathrm{H} \mid \mathrm{E})$ $=\mathrm{P}(\mathrm{H}) / \mathrm{P}(\mathrm{H})+\mathrm{P}(\neg \mathrm{H}) \times[\mathrm{P}(\mathrm{E} \mid \neg \mathrm{H}) / \mathrm{P}(\mathrm{E} \mid \mathrm{H})]$.

\begin{tabular}{|c|c|l|l|l|l|}
\hline intuitio & ISSN & Porto Alegre & Vol.11 $-\mathrm{N}^{\circ} .1$ & $\begin{array}{l}\text { Julho } \\
2018\end{array}$ & p.46-64 \\
\hline
\end{tabular}


O argumento do milagre comete a falácia da taxa-base? Apresentação, estado da arte e questões de formalização

considerações, assume-se, como indicado anteriormente, que uma probabilidade $x$ é grande se $x>0.85$, e $x$ é não-negligenciável se $x>0.2$. Caso considere que uma probabilidade $x$ é grande se $x>0.5$, basta $\mathrm{P}(\mathrm{H})=$ 0.1. Cremos, no entanto, que dificilmente um antirrealista será convencido por AMP se ele apenas concluir que $\mathrm{P}(\mathrm{V} \mid \mathrm{S})$ é pouco maior que 0.5. Parece-nos razoável admitir que um antirrealista seria convencido se $\mathrm{P}(\mathrm{V} \mid \mathrm{S})$ fosse, pelo menos, 0.9, o que exigiria que o valor de $\mathrm{P}(\mathrm{V})$ fosse ainda maior que 0.3. AMP leva, em suma, a um dilema claramente exposto por Sprenger (2016: 179; tradução nossa): ${ }^{9}$ “[...] AMP demonstra que (1) na medida que AM é valido, as suas premissas assumem inclinações realistas; (2) na medida em que AM é construído em premissas que são neutras entre o realista e o antirrealista, AM não consegue ser válido".

\section{Estado da arte em relação à crítica de Howson}

Como indicado, a crítica de Howson explicada despertou duas categorias de reações na literatura especializada: (A) supondo que AM comete a falácia da taxa-base, (A1) alguns autores propuseram abandonar AM enquanto um argumento a favor da tese epistemológica do realismo científico, ao passo que (A2) outros defenderam que apenas uma versão de AM comete a falácia da taxa-base, limitando, então, o escopo da crítica de Howson; (B) defendendo que AM não comete a falácia da taxa-base, (B1) certos autores alegaram alguns problemas na formalização probabilística de AM, (B2) enquanto outros buscaram refinar AMP de modo que a nova formalização evitasse a falácia em questão. Nesta seção, comentaremos brevemente tais reações - no apêndice 2 a este artigo, esquematizamo-las.

Howson (2013: 210-211) é um dos autores que propõe abandonarmos, em função de sua crítica, AM. Como a conclusão de AMP somente é derivada ao pressupor uma probabilidade prévia nãonegligenciável para " $T$ é aproximadamente verdadeira", AMP e AM não conseguem responder satisfatoriamente à questão sobre a verdade aproximada de uma teoria $T$.

Independentemente de Howson, Peter Lewis (2001) atribuiu a falácia da taxa-base a um dos principais argumentos para justificar posições antirrealistas: a indução pessimista. ${ }^{10} \mathrm{Em}$ posse da crítica de Howson e Lewis, P. D. Magnus \& Craig Callender (2003) defendem que ambos argumentos, apesar de

9 No original: "[...] the simple probabilistic model of the NMA demonstrates that (1) to the extent that the NMA is valid, its premises presuppose realist inclinations; (2) to the extent that the NMA builds on premises that are neutral between the realist and the anti-realist, it fails to be valid".

10 Em sua formulação original, o argumento da indução pessimista afirma que: "Havia muitas teorias no passado que (tanto quanto podemos dizer) eram genuinamente referenciais e empiricamente bem-sucedidas, as quais, não obstante, seríamos avessos a considerar como verdadeiras" (Laudan, 1984: 35). No original: "There were many theories in the past which (so far as we can tell) were both genuinely referring and empirically successful which we are nonetheless loathe to regard as approximately true".

\begin{tabular}{|c|c|l|l|l|l|}
\hline intuitio & ISSN & Porto Alegre & Vol.11 $-\mathrm{N}^{\circ} .1$ & $\begin{array}{l}\text { Julho } \\
2018\end{array}$ & p.46-64 \\
\hline
\end{tabular}


O argumento do milagre comete a falácia da taxa-base? Apresentação, estado da arte e questões de formalização

parecerem intuitivos, devem ser abandonados na disputa entre realistas e antirrealistas por possuírem apenas uma força retórica.

Cornelis Menke (2014), Leah Henderson (2015) e Richard Dawid \& Stephan Hartmann (2017) procuram, cada um a seu modo, mostrar que a crítica de Howson apenas incide em uma versão de AM. Ora, para tais autores, há duas versões de AM. AM pode referir-se apenas ao sucesso preditivo de uma teoria $T$ e concluir que ela é aproximadamente verdadeira (chamemos tal versão de AML, "argumento do milagre local"). AM pode igualmente referir-se ao sucesso de preditivo de um campo de pesquisa e concluir que ele é aproximadamente verdadeiro (AMG, "argumento do milagre global") - aliás, para Dawid \& Hartmann (2017), o sentido original de AM era AMG.

Como Howson expôs, AML comete a falácia da taxa-base. Segundo tais autores, porém, o mesmo não é o caso com AMG. Entender por que o mesmo não ocorre com AMG varia conforme cada um dos autores acima; indiquemos, então, apenas a ideia geral da proposta Dawid \& Hartmann (2017). Tais autores apresentam uma formalização de AMG, em que a observação $O$ (baseada em frequências) de que $x$ de um total de $y$ teorias de uma área de pesquisa $A$ tiveram sucesso preditivo aumenta a probabilidade de que uma teoria $T$ em $A$ seja aproximadamente verdadeira, dependendo apenas da probabilidade do sucesso preditivo de $T$ dado $O$ e da verdade aproximada de $T$ dado $O$. Não se recorre, portanto, à probabilidade prévia de $T$.

Os autores acima assumem que a formalização probabilística do argumento do milagre (AMP) é satisfatória. Como indicado, essa crença foi atacada de dois modos: certos autores alegaram alguns problemas na formalização probabilística de AM, enquanto outros buscaram refinar AMP de modo que a nova formalização evitasse a falácia em questão.

Jan Sprenger (2016) considera, em particular, que AMP é uma formalização fraca de AM. Para este autor, uma formalização de AM deve considerar não somente o impacto do sucesso preditivo e explicativo de uma teoria $T$, mas também a estabilidade de teorias científicas. Formalizando AM com esse novo elemento e levando-se em conta contextos de estabilidade de teorias científicas, Sprenger pretende mostrar como tal estabilidade e o sucesso preditivo de uma teoria $T$ são fortes evidências de que ela é empiricamente adequada - por motivos teóricos que não podemos comentar aqui, Sprenger prefere não adotar "aproximadamente verdadeira".

Por fim, Stathis Psillos (2009) apresenta uma série de objeções à crítica de Howson a AM. Curiosamente, Howson (2013) apenas replica uma versão apresentada por Psillos de AM em que não se recorre a probabilidades prévias. Psillos, contudo, igualmente expõe objeções relacionadas à formalização de AM suposta na crítica em questão. Comentemos apenas duas delas.

Psillos (ibid:: 61-62) argumenta que, supondo que taxas-base não equivalham a probabilidades prévias, taxas-base para a verdade ou falsidade de uma teoria dependem de como determinamos o número

\begin{tabular}{|c|c|l|l|l|l|}
\hline intuitio & $\begin{array}{c}\text { ISSN } \\
1983-4012\end{array}$ & Porto Alegre & Vol.11- No.1 & $\begin{array}{l}\text { Julho } \\
2018\end{array}$ & p.46-64 \\
\hline
\end{tabular}


O argumento do milagre comete a falácia da taxa-base? Apresentação, estado da arte e questões de formalização

relevante de teorias. Ora, para fazê-lo, utilizamos determinado conceito de sucesso preditivo que tanto pode aumentar quanto diminuir o número de teorias falsas e verdadeiras. Outra crítica a AMP sustenta que ele é ambíguo. Com efeito, Psillos (ibid.: 66) afirma que, se ele se refere a uma teoria $T$ específica (e.g., a teoria da relatividade geral de Einstein) para a qual há informações históricas abundantes de que ela é aproximadamente verdadeira, não recorremos (nem os cientistas) à taxa-base de teorias verdadeiras para afirmar que $T$ é aproximadamente verdadeira. Mas, se ele se refere a uma teoria $T$ qualquer, neste caso sim precisamos recorrer à taxa-base de teorias verdadeiras.

Por ter motivado o estado da arte descrito, parece razoável defender que a formalização probabilística do argumento do milagre, AMP, é fecunda. Com efeito, além de AMP se dirigir a um argumento importante em filosofia informal, atribuindo-lhe uma crítica relativamente nova, o debate em torno de AMP também pode levar em conta, como mostra Psillos, se AMP é uma boa formalização de AM. Por discutir novos aspectos de assuntos já discutidos em filosofia informal e assuntos que não eram discutidos, mas que possuem interesse filosófico, ou seja, levando-se em conta a fecundidade de AMP, podemos, na esteira de Hansson (2000: 173), afirmar que AMP é uma formalização razoável de AM.

Ainda assim, um realista pode, junto a Psillos, se sentir insatisfeito com AMP e com todo o debate dele derivado por outro motivo: como toda formalização, AMP distancia-se de AM realizando algumas idealizações. Um realista estaria, nesse caso, preocupado com o critério de similaridade de uma formalização. No contexto de uma análise sobre uma formalização específica do argumento ontológico a favor da existência de Deus, Ricardo Silvestre (2015: 159) parece ter em mente o critério de similaridade ao afirmar que, se o afastamento de uma formalização em relação a seu objeto-alvo é grande e não justificado, é penoso reconhecer o argumento formal (no nosso caso, AMP) como uma formalização do argumento original (AM). Em posse dessa observação de Silvestre, tentaremos investigar, na próxima seção, se é ou não o caso de que o afastamento de AMP é demasiado em relação ao AM. ${ }^{11}$

\section{AMP e a inferência à melhor explicação}

Para investigar se AMP afasta-se muito de AM, teremos como foco de nossas considerações um dos elementos considerados cruciais em AM: a sua natureza de inferência à melhor explicação (IME). ${ }^{12} \mathrm{~A}$

11 Ambos critérios apresentados são, claramente, vagos e passíveis de objeções robustas. Contudo, eles foram os únicos critérios encontrados na literatura metafilosófica sobre como avaliar formalizações em filosofia. A carência de um maior número de investigações a esse respeito ao mesmo tempo em que se considera a disseminação de instrumentos formais em filosofia motiva, claramente, o desenvolvimento de melhores critérios. Para este artigo, énos suficiente, porém, aqueles apresentados acima.

12 Musgrave (1988: 237) é, talvez, um dos primeiros a relacionar AM a IME e também à abdução. Embora alguns autores utilizem inferência à melhor explicação e abdução como termos intercambiáveis, não iremos fazê-lo.

\begin{tabular}{|c|c|c|c|c|c|}
\hline intuitio & $\begin{array}{c}\text { ISSN } \\
1983-4012\end{array}$ & Porto Alegre & Vol.11 - N $\mathrm{N}^{\mathrm{o}} .1$ & $\begin{array}{l}\text { Julho } \\
2018\end{array}$ & p.46-64 \\
\hline
\end{tabular}


O argumento do milagre comete a falácia da taxa-base? Apresentação, estado da arte e questões de formalização

questão desta seção pode ser, então, formulada do seguinte modo: o aparato Bayesiano suposto em AMP consegue acomodar a natureza de inferência à melhor explicação de AM? Psillos $(2009 ; 2007)$ investigou a relação entre IME e o Bayesianismo sem, porém, relacionar suas considerações com AM e AMP. Iremos, não obstante, comentar as suas observações por dois motivos: primeiro, pois parece-nos simples relacioná-las a AM e AMP; segundo, porque Psillos, embora responda negativamente à proposta de acomodar IME no aparato Bayesiano, discute autores que respondem afirmativamente (por exemplo, Lipton, 2004).

Sem adentrar a detalhes específicos, nem na sua história, IME é um gênero de inferência pelo qual pode-se, a partir da premissa de que uma hipótese $H$ explica, se verdadeira, determinados dados $D$ e de que não há nenhuma outra hipótese que explique $D$ tão bem quanto $H$, concluir que $H$ é provavelmente verdadeira (Psillos, 2007: 442). AM procederia, então, conforme IME:

\section{(IME) Inferência para a melhor explicação}

P1: $\quad D$ é uma coleção de fatos e observações.

P2: $\quad H$ explica, se verdadeira, $D$.

P3: $\quad$ Nenhuma outra hipótese explica $D$ tão bem quanto $H$.

Concl.: $H$ é (provavelmente) verdadeira.

\section{(AM*) Argumento do Milagre conforme IME}

P1*: A teoria $T$ é bem-sucedida preditivamente (D).

P2*: $T$ ser aproximadamente verdadeira (H) explica, se verdadeira, $D$.

P3*: Nenhuma hipótese explica $D$ tão bem quanto $T$.

Concl.: $T$ é aproximadamente verdadeira.

Esquema 2: Comparação entre IME e AM*.

Psillos (2009: 195; 2007) defende que IME é uma regra que permite aceitar $H$. Ora, em AMP teríamos apenas que $T$ é bem provável condicional em seu sucesso preditivo. O autor reconhece - embora de maneira breve e sem maiores considerações - que um Bayesiano poderia adotar algum princípio que lhe permita concluir que, se a probabilidade de $H$ é maior a algum limiar $x$, então um agente pode aceitar

Conforme veremos nas considerações finais, a inferência abdutiva, formulada por Charles Sanders Peirce (CP 5.189), possui elementos extremamente peculiares de modo que, para ele, até a Natureza realiza abduções (IBRI, 2015: 67). Sobre a não-equivalência entre a inferência à a melhor explicação e a abdução Peirceana, ver Campos (2011).

\begin{tabular}{|c|c|c|c|c|c|}
\hline intuitio & $\begin{array}{c}\text { ISSN } \\
1983-4012\end{array}$ & Porto Alegre & Vol.11- $\mathrm{N}^{\circ} .1$ & $\begin{array}{l}\text { Julho } \\
2018\end{array}$ & p.46-64 \\
\hline
\end{tabular}


O argumento do milagre comete a falácia da taxa-base? Apresentação, estado da arte e questões de formalização

racionalmente $H$ (tese Lockeana), mas tais princípios seriam demais problemáticos - talvez em virtude de paradoxos como o paradoxo da loteria. ${ }^{13}$

Outra crítica de Psillos (2009: 200) afirma que IME é ampliativa no sentido de permitir concluir hipóteses que excedem os dados que as baseiam, ao passo que o aparato Bayesiano suposto em AMP, conforme inclusive Howson (2000: 177; tradução nossa) ${ }^{14}$ admite, "[...] não produz novo conteúdo factual". Psillos (2007: 446-447) reconhece que um Bayesiano poderia atribuir probabilidades prévias maiores a hipóteses ampliativas e, então, calcular sua probabilidade posterior. Isso, no entanto, seria atribuir a IME apenas um papel no contexto de descoberta e retirar dela seu papel no contexto de justificação que, para Psillos, é-lhe essencial.

Por fim, Psillos (2009: 196-200) argumenta que, embora o aparato Bayesiano possa acomodar elementos explicativos, como Lipton indica, há razões para não querermos fazê-lo. Conforme Psillos, ou elementos explicativos (i) seriam expressos pela razão $(\mathrm{P}(\mathrm{E} \mid \neg \mathrm{H}) / \mathrm{P}(\mathrm{E} \mid \mathrm{H})$ ), ou (ii) pela atribuição de maior probabilidade prévia a boas hipóteses explicativas.

Se (i), conforme a falácia da taxa-base mostra, tal razão é quase sempre insuficiente para determinar a probabilidade posterior de uma hipótese $H$. Quase sempre pois, se apenas a hipótese $H$ explica a evidência $E$, então $\mathrm{P}(\mathrm{E} \mid \neg \mathrm{H})=0$; se $\mathrm{P}(\mathrm{E} \mid \neg \mathrm{H})=0$, conforme a versão do teorema de Bayes da nota de rodapé 9, $\mathrm{P}(\mathrm{H} \mid \mathrm{E})=\mathrm{P}(\mathrm{H}) / \mathrm{P}(\mathrm{H})=1$. Em face disso, conclui Psillos (2009: 198; tradução nossa): ${ }^{15}$ "Mas isso mostra que IME é acomodada dentro do Bayesianismo? De certa forma, sim; não obstante, essa forma não é muito excitante. Se houvesse apenas uma explicação potencial, seria tolice não aceitá-la”.

Se (ii), então um Bayesiano subjetivo poderia muito bem atribuir probabilidades prévias maiores a boas hipóteses explicativas. Isso, porém, apenas significa uma permissão para que probabilidades prévias sejam reguladas por fatores explicativos, mas não que elas devam fazê-lo. Se assumimos que elas devam ser reguladas por fatores explicativos, teríamos que defender um princípio que restrinja as probabilidades prévias. Assim, sairíamos de um Bayesianismo subjetivo e defenderíamos alguma forma de Bayesianismo

13 O paradoxo da loteria pode ser expresso como a seguir. Suponha quatro condições: (i) há uma loteria justa vendendo 100 bilhetes, numerados de 1 a 100; (ii) 1 bilhete será sorteado e os outros 99 não serão sorteados; (iii) se o grau de crença em uma proposição excede 0.95 , um agente racional A deve aceitá-la, e (iv) se A aceita $\mathrm{H}_{1}$ e aceita $\mathrm{H}_{2}$, então A deve aceitar a conjunção de $\mathrm{H}_{1}$ e $\mathrm{H}_{2}$. Em face a essas informações, parece razoável supor que o grau de crença de A em "o bilhete 1 não será sorteado" possui valor 0.99 , assim como para o bilhete 2 e assim por diante. Dado (iii), o agente deveria aceitar que "o bilhete 1 não será sorteado", assim como que "o bilhete 2 não será sorteado", etc. Contudo, em função de (iv), A aceitaria que "o bilhete 1 não será sorteado $e$ o bilhete 2 não será sorteado... e o bilhete 100 não será sorteado", o que lhe é, altamente, contra-intuitivo, pois A sabe que, por (ii), exatamente um bilhete será sorteado. Tem-se, assim, um paradoxo: A teria que aceitar que nenhum bilhete será sorteado mesmo sabendo que um será.

14 No original: "[...] does not beget new factual content".

15 No original: "But does it show that IBE is accommodated within Bayesianism? In a sense, it does, and yet this sense is not terribly exciting. If there was only one potential explanation, it would be folly not to accept it."

\begin{tabular}{|c|c|l|l|l|l|}
\hline intuitio & $\begin{array}{c}\text { ISSN } \\
1983-4012\end{array}$ & Porto Alegre & Vol.11- $\mathrm{N}^{\mathrm{o}} .1$ & $\begin{array}{l}\text { Julho } \\
2018\end{array}$ & p.46-64 \\
\hline
\end{tabular}


objetivo que, segundo Psillos (2009: 199), poucas pessoas adotariam. Em suma, seríamos levados a um dilema (ibid.; tradução nossa): ${ }^{16}$

A maneira como descrevi as coisas leva-nos a um dilema. Ou acomodar (de modo relativamente fácil) IME no Bayesianismo, mas perder o entusiasmo e a maior parte da força putativa de IME, ou acomodar uma versão interessante de IME, mas modificando radicalmente o Bayesianismo. Acho que todos concordamos que o Bayesianismo é a melhor teoria de confirmação disponível. Contudo, pelo menos alguns de nós não estão dispostos a pensar que o Bayesianismo é a palavra final sobre o assunto, uma vez que pensamos que há mais na racionalidade (e no método científico) do que os Bayesianos permitem (Psillos, 2009: 199).

As objeções acima de Psillos quanto à capacidade do aparato Bayesiano acomodar IME - e, em nosso contexto, AMP ser uma formalização razoável de AM, levando-se em conta o critério de similaridade - não nos parecem decisivas por três motivos. Em primeiro lugar, ainda que as regras que permitam conectar a probabilidade de alguma proposição $P$ com sua aceitação racional enfrentem dificuldades, não se segue, simplesmente por isso, que esse é um projeto condenado ao fracasso e que, então, jamais AMP acomodaria o caráter de aceitação de IME. Em segundo lugar, criticar um Bayesiano subjetivo pelo fato de ele apenas permitir que boas explicações tenham maiores probabilidades prévias e não que elas devam fazê-lo é, salvo melhor juízo, exigir que um aparato formal resolva disputas para as quais ele não foi desenvolvido. Em terceiro lugar, mesmo admitindo que seja verdade a afirmação de que poucos autores adotariam um Bayesianismo objetivo com elementos explicativos, isso de modo nenhum implica uma falha de tal aparato em acomodar o que quer que seja.

Em poucas palavras, parece-nos que as objeções de Psillos, quando inseridas no contexto da formalização de AM por AMP, não mostram um afastamento demasiado da formalização em relação ao argumento original. Em verdade, talvez o único grande afastamento realizado por AMP seja traduzir, sem justificação, P(A | B) por quão bem $B$ explica $A$. Ora, caso utilizemos probabilidades condicionais, supondo o cálculo Kolmogoroviano, como um modelo de explicação, algumas graves dificuldades irão, certamente, aparecer. Por exemplo, (i) se $A$ é uma tautologia e $B$ qualquer explicação, tem-se que P(A | B) = 1 ; (ii) se $B$ é uma explicação qualquer para uma evidência $A$ qualquer, mas $\mathrm{P}(\mathrm{B})=0$, então $\mathrm{P}(\mathrm{A} \mid \mathrm{B})$ é indefinida. ${ }^{17}$ Não obstante, no contexto da discussão sobre AM e AMP, cremos que dificilmente tais situações ocorreriam: (i) realistas científicos interessam-se por teorias contingentes, $\log$ o P(A) jamais será 1, e (ii) por teorias maduras, ou seja, com alto grau de sucesso preditivo de modo que $\mathrm{P}(\mathrm{B})$ é sempre maior

16 No original: "The way I have described things leads us to a dilemma. Either accommodate (relatively easily) IBE within Bayesianism but lose the excitement and most of the putative force of IBE or accommodate an interesting version of IBE but radically modify Bayesianism. I guess we all agree that Bayesianism is the best theory of confirmation available. But at least some of us are unwilling to think that Bayesianism is the final word on the matter, since we think that there is more to rationality (and to scientific method) than Bayesians allow.".

17 É importante assinalar que essa objeção pode ser neutralizada à medida que se assume algum modelo probabilístico em que probabilidades condicionais são definidas mesmo se o valor do denominador for 0 .

\begin{tabular}{|c|c|l|l|l|l|}
\hline intuitio & ISSN & Porto Alegre & Vol.11 $-\mathrm{N}^{\circ} .1$ & $\begin{array}{l}\text { Julho } \\
2018\end{array}$ & p.46-64 \\
\hline
\end{tabular}


O argumento do milagre comete a falácia da taxa-base? Apresentação, estado da arte e questões de formalização

que zero. Assim, AMP é, salvo melhor juízo, uma formalização razoável de AM, levando-se em conta sua natureza de inferência à melhor explicação.

\section{Considerações finais}

Neste artigo, expusemos uma crítica recente ao argumento do milagre (AM) dada pelo Bayesiano Colin Howson $(2000 ; 2013)$. Formalizando o argumento do milagre probabilisticamente, Howson mostrou como sua conclusão se segue apenas se assumirmos uma probabilidade prévia não-negligenciável para a verdade aproximada de uma teoria $T$.

Descrevemos, em seguida, a reação da literatura especializada em relação a ela. Conforme exposto, tal reação divide-se em duas categorias. Por um lado, autores que concordam com a diagnóstico de Howson ou propuseram abandonar AM ou defenderam que uma versão global do argumento do milagre (AMG) não comete a falácia da taxa-base. Por outro lado, defendendo que AM não comete a falácia da taxa-base, outros autores alegaram problemas na formalização probabilística de AM, ao passo que outros buscaram refiná-la de modo a evitar a falácia em questão.

Por fim, investigamos se a formalização probabilística do argumento do milagre (AMP), suposta no debate em questão, é razoável. Adotando o critério de fecundidade, concluímos, em face ao estado da arte exposto, que sim. Adotando o critério de similaridade e supondo que AM instancie uma inferência à melhor explicação, concluímos igualmente que sim, porque alguma das idealizações de AMP podem ser contornadas ou não são graves no debate em que está inserida. Logo, AMP satisfaz tanto o critério de fecundidade quanto o de similaridade de uma formalização.

Em face ao exposto, parece irresistível afirmar que a crítica de Howson ao argumento do milagre é decisiva. Gostaríamos, contudo, de sugerir rapidamente nestas considerações finais uma limitação que ela sofre. Quando investigamos se AMP acomodava a inferência suposta em AM, assumimos que se tratava de uma inferência à melhor explicação (IME). Queremos indicar nos próximos parágrafos que se assumirmos que a inferência de AM é, na verdade, uma abdução no sentido Peirceano, então AMP não é uma formalização razoável de AM. Tais considerações nos levarão a concluir que: se a inferência de AM é entendida como uma IME, a crítica de Howson é razoável tanto segundo o critério de fecundidade quanto segundo o critério de similaridade; se, porém, a inferência de AM é entendida como uma abdução, a crítica de Howson deixa de ser decisiva.

Embora o conceito de abdução varie ao longo da obra de Peirce, sua característica principal é não somente a geração de hipóteses que expliquem fatos surpreendentes, como a razoabilidade delas em função de seu poder explicativo (CP 5.189). Acompanhando as considerações de Psillos (2011) sobre a

\begin{tabular}{|c|c|l|l|l|l|}
\hline intuitio & $\begin{array}{c}\text { ISSN } \\
1983-4012\end{array}$ & Porto Alegre & Vol.11- $\mathrm{N}^{\mathrm{o}} .1$ & $\begin{array}{l}\text { Julho } \\
2018\end{array}$ & p.46-64 \\
\hline
\end{tabular}


O argumento do milagre comete a falácia da taxa-base? Apresentação, estado da arte e questões de formalização

abdução Peirceana, haveria pelo menos dois modos por meio dos quais o pragmaticista relacionava tal argumento e probabilidade.

Por meio do primeiro modo, Peirce (CP 7.202; tradução nossa) ${ }^{18}$ parece afirmar que a hipótese a ser adotada ao fim de uma inferência abdutiva pode ser provável em si mesma: "Uma hipótese, então, tem de ser adotada, a qual é provável em si mesma e torna os fatos prováveis". Interpretando "provável em si mesma" como uma probabilidade prévia não-negligenciável a determinada hipótese, a própria estrutura de abdução já assumiria justamente o que a falácia da taxa-base condena. Portanto, atribuir a falácia da taxabase ao AMP apenas ressaltaria uma de suas propriedades e não teria, por conseguinte, efeito nenhum.

Por meio do segundo modo, Peirce, ao relacionar a inferência abdutiva com os demais tipos de inferências, ${ }^{19}$ afirma explicitamente que probabilidade não assume nenhum papel na abdução. Conforme resume Psillos (2011: 139-143; tradução nossa): ${ }^{20}$ "O que tudo isso quer dizer é que a inferência abdutiva não é, por si mesma, o tipo de inferência que pode ou leva a conclusões prováveis. [...] A abdução pode conferir plausibilidade ou razoabilidade a suas conclusões, mas a probabilidade delas é determinada por seus testes adicionais". Assumindo as considerações acima, não somente atribuir a falácia da taxa-base a AMP não faria sentido, como também qualquer tentativa de formalizá-lo probabilisticamente não faria sentido.

Por fim, notamos que a conclusão de uma abdução é provisória, ou seja, aceita-se provisoriamente que uma hipótese explique determinados fatos. Em virtude de novas investigações, isso pode não ser mais o caso e, então, uma nova hipótese deverá ser formulada. Cremos que AMP não acomoda tal característica da abdução mesmo adotando-se os princípios comentados por Psillos na seção 4. Em suma, se entendermos que a inferência de AM é uma abdução em vez de IME, parece-nos que a crítica de Howson $(2000 ; 2013)$ deixa de ser robusta não simplesmente por AMP afastar-se em relação a AM, mas principalmente por inferências abdutivas ou suporem probabilidades prévias consideráveis a suas hipóteses ou dispensarem o uso de probabilidade na sua construção.

\section{Agradecimentos}

À André Neiva, Danilo Dantas, Isadora Sancassani e João Moraes pela revisão e valiosos comentários nas primeiras versões deste artigo. À Fundação de Amparo à Pesquisa do Estado de São

18 No original: "A hypothesis then, has to be adopted, which is likely in itself, and renders the facts likely".

19 Peirce as relaciona da seguinte maneira: (i) uma hipótese é formulada para explicar fatos contrários a expectativas prévias: abdução (CP 7.202); (ii) em seguida, deduz-se suas consequências experimentais menos prováveis: dedução (CP 7.203); (iii) depois, realizam-se experimentos que, ou darão suporte à hipótese, ou a refutarão demandando a formulação de outra hipótese: indução (CP 7. 206).

20 No original: "What all this means is that abductive inference per se is not the kind of inference that can or does lead to likely conclusions. [...] Abduction might confer plausibility or reasonableness on its conclusion, but their probability is determined by their further testing".

\begin{tabular}{|c|c|l|l|l|l|}
\hline intuitio & $\begin{array}{c}\text { ISSN } \\
1983-4012\end{array}$ & Porto Alegre & Vol.11- $\mathrm{N}^{\mathrm{o}} .1$ & $\begin{array}{l}\text { Julho } \\
2018\end{array}$ & p.46-64 \\
\hline
\end{tabular}


O argumento do milagre comete a falácia da taxa-base? Apresentação, estado da arte e questões de formalização

Paulo pela bolsa de mestrado (Processo $N^{\circ}$ 2016/03251-2) que favoreceu o desenvolvimento da pesquisa aqui exposta.

\section{Referências}

CAMPOS, D. On the Distinction between Peirce's Abduction and Lipton's Inference to The Best Explanation. Synthese. vol. 180, n. 3, p. 419-42, 2011.

CASSCELLS, W., SCHOENBERGER, A., GRAYBOYS, T. Interpretation by Physicians of Clinical Laboratory Results. New England Journal of Medicine. vol. 299, p. 999-1001, 1978.

DAWID, R. \& HARTMANN, S. The No Miracles Argument without The Base Rate Fallacy. Synthese, Open Access. Último acesso em: 03 de maio de 2018, <https://doi.org/10.1007/s11229-017-1408-x>, 2017.

EASWARAN, K. Bayesianism I: Introduction and Arguments in Favor. Philosophy Compass, vol. 6, n. 5, p. $312-$ 320, 2011.

HANSSON, S.O. Formalisation in Philosophy. Bulletin of Symbolic Logic, vol. 6. n. 2, p. $162-175,2000$.

HENDERSON, L. The No-Miracles Argument and The Base Rate Fallacy. Synthese, vol. 194, n. 4, p. 1295-1302, 2017.

HOWSON, C. Hume's Problem: Induction and the Justification of Belief. New York: Oxford University Press, 2000. . Exhuming The No Miracles Argument. Analysis, vol. 73, n.2, p. 205-211, 2013.

IBRI, I. A. Kósmos Noetós: a Arquitetura Metafísica de Charles S. Peirce. São Paulo: Paulus, 2015.

KOLMOGOROV, A. N. Foundations of the Theory of Probability. Tradução de Nathan Morrison. New York: Chelsea Publishing Company, 1950.

LAUDAN, L. A Confutation of Convergent Realism. IN: LEPLIN, J. (Ed.). Scientific realism. Berkeley: University of California Press, 1984, p. 218-49.

LEWIS, P. Why The Pessimistic Induction is a Fallacy. Synthese, vol. 129, p. 371-380, 2001.

LIPTON, P. Inference to The Best Explanation. $2^{a}$ ed. London: Routledge, 2004.

MAGNUS, P. D., \& CALLENDER, C. Realist Ennui and The Base Rate Fallacy. Philosophy of Science, vol. 71, p. 320-338, 2003.

MENKE, C. Does The Miracles Argument embody a Base Rate Fallacy? Studies in History and Philosophy of Science, vol. 45, p. 103-108, 2014.

MUSGRAVE, A. The Ultimate Argument for Scientific Realism. IN: NOLA, R. (Ed.). Relativism and Realism in Science. Dordrecht: Kluwer Academic Publishers, 1988.

NEIVA, A. Probabilismo e Bayesianismo em Epistemologia. PERI, v. 07, n. 02, p. 45-69, 2015.

PEIRCE, C. S. Collected Papers of Charles Sanders Peirce, Vols. 1-8. In: WEISS, P. [et al.] (Eds.). Cambridge: Harvard University Press, 1932 - 1958.

PSILLOS, S. Scientific Realism: How Science Tracks Truth. London: Routledge. 1999.

. The Fine Structure of Inference to The Best Explanation. Philosophy and Phenomenological Research, vol. 74, n. 2, p. 441-448, 2007.

\section{9.}

Knowing The Structure of Nature: Essays on Realism and Explanation. New York: Palgrave McMillan,

An Explorer Upon Untrodden Ground: Peirce on Abduction. In: GABBAY, D. \& WOODS, J. (Eds.) Handbook of the History of Logic: Volume 10 - Inductive Logic. Elsevier: Amsterdam 2011.

PUTNAM, H. Mathematics, Matter and Method. Cambridge: Cambridge University Press, 1975.

RESCORLA, M. Chrysippus' Dog as a Case Study in Non-Linguistic Cognition. IN: LURZ, R. (ed.). The Philosophy of Animal Minds. New York: Cambridge, 2009, p. 52-71.

Bayesian Perceptual Psychology. IN: MATTHEN, M. (Ed.). The Oxford Handbook of the Philosophy of Perception. Oxford: Oxford University Press, 2015, p. 694-716.

SERIĖS, P. Le Cerveau est-il une Machine Bayésienne? IN: DROUET, I. (Dir.). Le Bayésianisme aujourd'hui. Paris: Éditions Matériologiques, 2016.

SILVESTRE, R. S. On The Logical Formalization of Anselm's Ontological Argument. Revista Brasileira de Filosofia da Religião, vol. 2, n .2, p 142-16, 2015.

SPRENGER, J. The Probabilistic No Miracles Argument. European Journal for Philosophy of Science, vol. 6, p. 173-189, 2016.

\begin{tabular}{|c|c|l|l|l|l|}
\hline intuitio & $\begin{array}{c}\text { ISSN } \\
1983-4012\end{array}$ & Porto Alegre & Vol.11- $\mathrm{N}^{\mathrm{o}} .1$ & $\begin{array}{l}\text { Julho } \\
2018\end{array}$ & p.46-64 \\
\hline
\end{tabular}


O argumento do milagre comete a falácia da taxa-base? Apresentação, estado da arte e questões de formalização

VAN FRAASSEN, B.C. The Scientific Image. Oxford: Clarendon Press, 1980.

WEISBERG, J. Varieties of Bayesianism. IN: GABBAY, D.; HARTMANN, S.; WOODS, J. (Eds.). Handbook of the History of Logic, vol. 10, Inductive Logic. Amsterdan: North-Holland, 2011, p. 477-551.

WORRALL, J. Structural Realism: The Best of Both Worlds. Dialectica. vol. 43. n 1-2, p. 99-124, 1989.

\begin{tabular}{|l|c|c|c|c|c|}
\hline intuitio & $\begin{array}{c}\text { ISSN } \\
1983-4012\end{array}$ & Porto Alegre & Vol.11- No.1 & $\begin{array}{c}\text { Julho } \\
2018\end{array}$ & p.46-64 \\
\hline
\end{tabular}




\section{Apêndice 1 - Contínuo de posições bayesianas}

GRAD $\wedge$ PROB $\wedge$ RC
Bayesianismo subjetivo
$\begin{aligned} & \text { Legenda: GRAD (gradualismo), PROB (probabilismo), RC (revisão pela condicionalização), PP (princípio principal), PPE (princípio principal } \\ & \text { estatístico), PI (princípio da indiferença), PME (princípio de máxima entropia), PS (princípio da simplicidade). }\end{aligned}$

\section{Apêndice 2 - Estado da arte em relação à crítica de Howson}

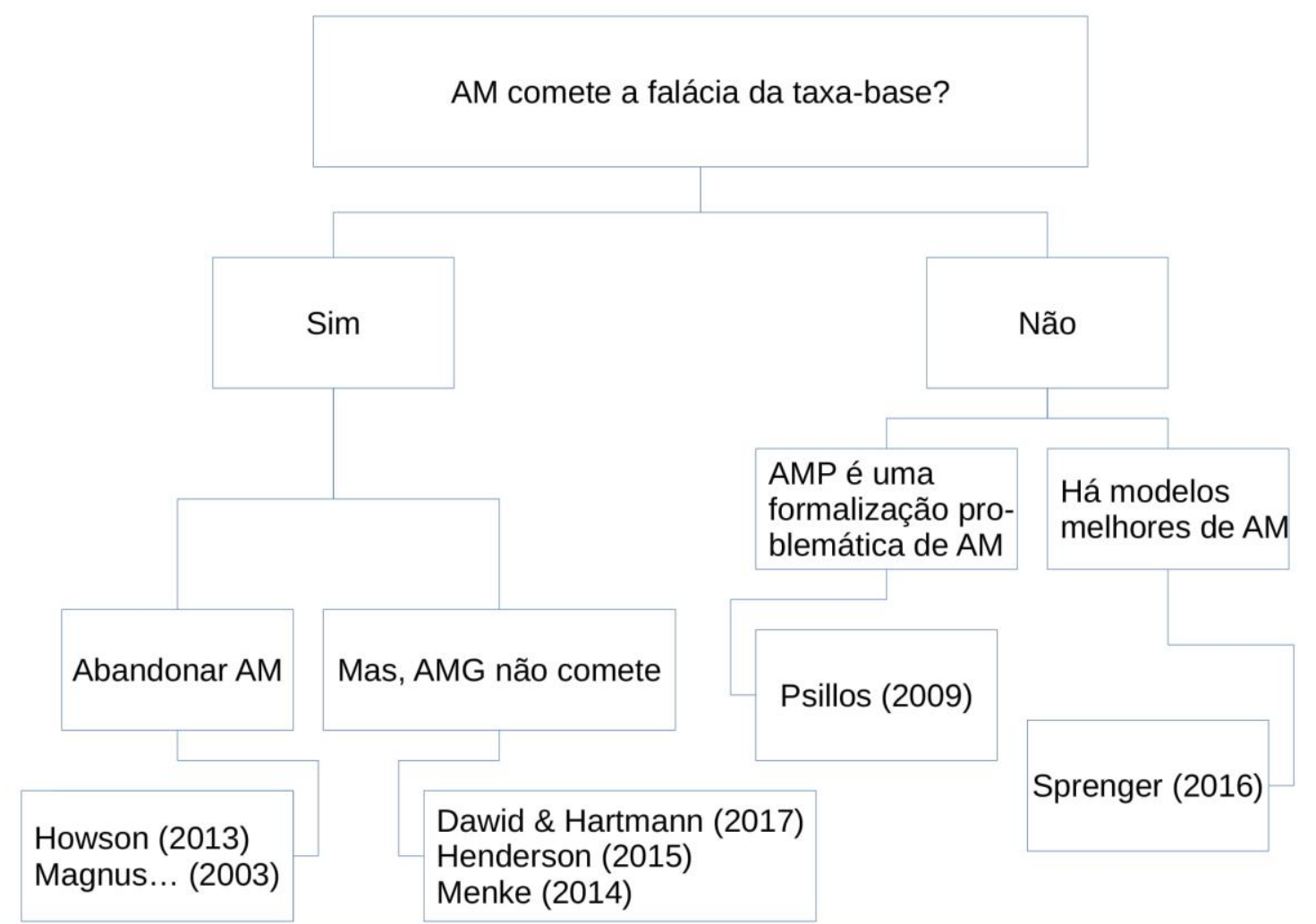

Legenda: AM (argumento do milagre), AMP (argumento do milagre probabilístico) e AMG (argumento do milagre global)

\begin{tabular}{|c|c|l|l|l|l|}
\hline intuitio & $\begin{array}{c}\text { ISSN } \\
1983-4012\end{array}$ & Porto Alegre & Vol.11- $\mathrm{N}^{\mathrm{o}} .1$ & $\begin{array}{l}\text { Julho } \\
2018\end{array}$ & p.46-64 \\
\hline
\end{tabular}

